\title{
Abnormal radial artery in Down's syndrome
}

\author{
R N S LO, M P LEUNG, K C LAU, AND C Y YEUNG \\ Department of Paediatrics, Queen Mary Hospital, University of Hong Kong
}

SUMmARY Abnormal arterial patterns in the forearm were discovered in 12 of 77 patients (16\%) with Down's syndrome. Eight patients had an enlarged anterior interosseous artery, the distal portion of which was palpable over the dorsum of the hand; in seven it was associated with absence of the radial artery and in one it coexisted with the radial and ulnar arteries. Three patients had a hypoplastic radial artery and a relatively dominant ulnar artery. One patient had a vestigial radial artery that ended as muscular branches in the forearm. The developmental aspects of these aberrations are discussed.

The radial artery in its superficial position on the volar aspect of the wrist offers a well known access for clinical evaluation of the circulation. Its palpable pulsation on the radial side of the wrist between the flexor carpi radialis tendon medially and the bony prominence of the lower part of the radius laterally has been remarkably constant, and reports on variations are scanty. ${ }^{12}$ Reports on congenital heart diseases in Down's syndrome are ample $;^{3-8}$ extracardiac vascular abnormalities, however, have been infrequently described, and radial artery aberrations have not been mentioned. Recent detection of two patients, both with Down's syndrome and congenital heart disease, in whom the radial artery pulsation was not palpable in the usual position over the wrist, prompted us to look at the incidence of this phenomenon.

\section{Patients and methods}

From January 1983 to December 1985 a total of 1087 Chinese children were studied. Of these, 510 were otherwise normal children with congenital heart disease and 77 were patients with Down's syndrome ( 40 boys and 37 girls), of whom 50 had congenital heart disease. Five hundred normal children without heart disease were included as controls.

Each patient was examined independently by at least two paediatricians for the presence of abnormal arterial pulsations in the hand. The radial and ulnar arteries were carefully palpated for on the flexor surface of the wrist and distal forearm. Patients with previous cannulation of either artery were excluded. The radial or ulnar artery was considered abnormal if (a) its pulsation was not

Table Abnormality of the radial artery and associated congenital heart disease in 12 patients with Down's syndrome

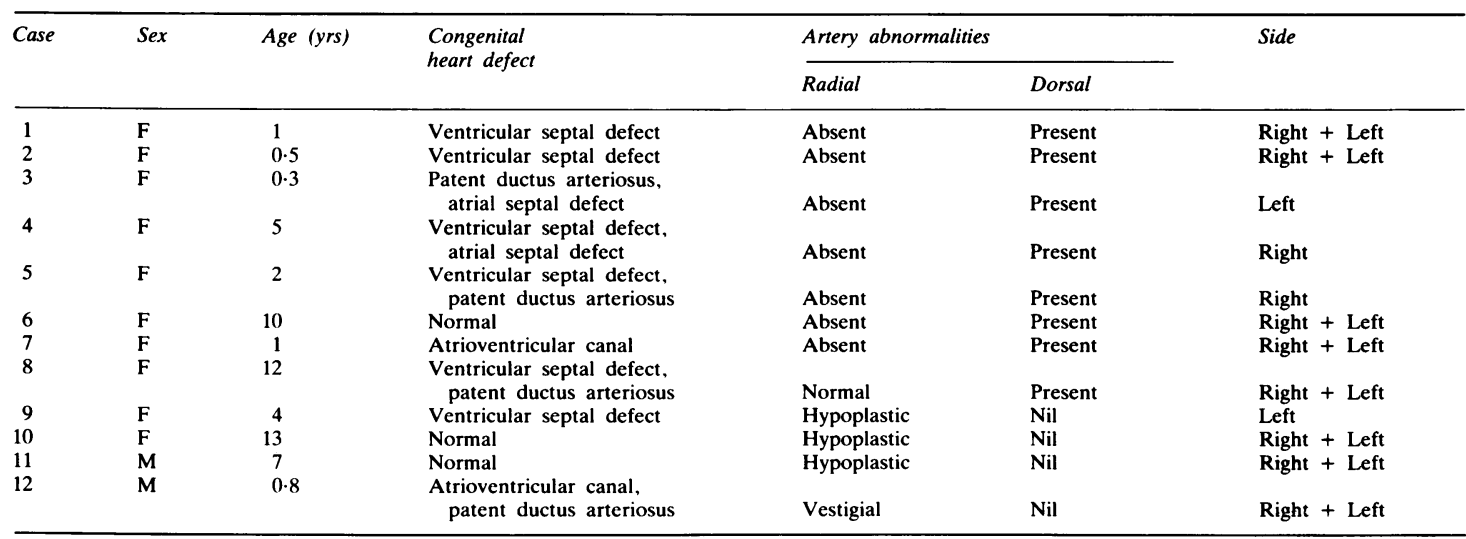


palpable and no arterial flow could be recorded with a Doppler flow detector in its usual position at the wrist, or (b) the radial pulsation was notably weaker than that of the ulnar artery. Presence of an additional arterial pulsation apart from that of the radial or ulnar artery in another position, particularly over the dorsum of the wrist, was carefully looked for. The cardiac anatomy in those with congenital heart disease was defined by cardiac catheterisation or two dimensional echocardiography, or both. In patients with abnormal arterial pulses the arterial pattern of the forearm was visualised by contrast injection into the subclavian or brachial artery. The subclavian artery was entered through antegrade or retrograde catheterisation of the aorta during cardiac catheterisation.

The brachial artery was cannulated by percutaneous puncture in one patient with atrioventricular canal defect and patent ductus arteriosus before

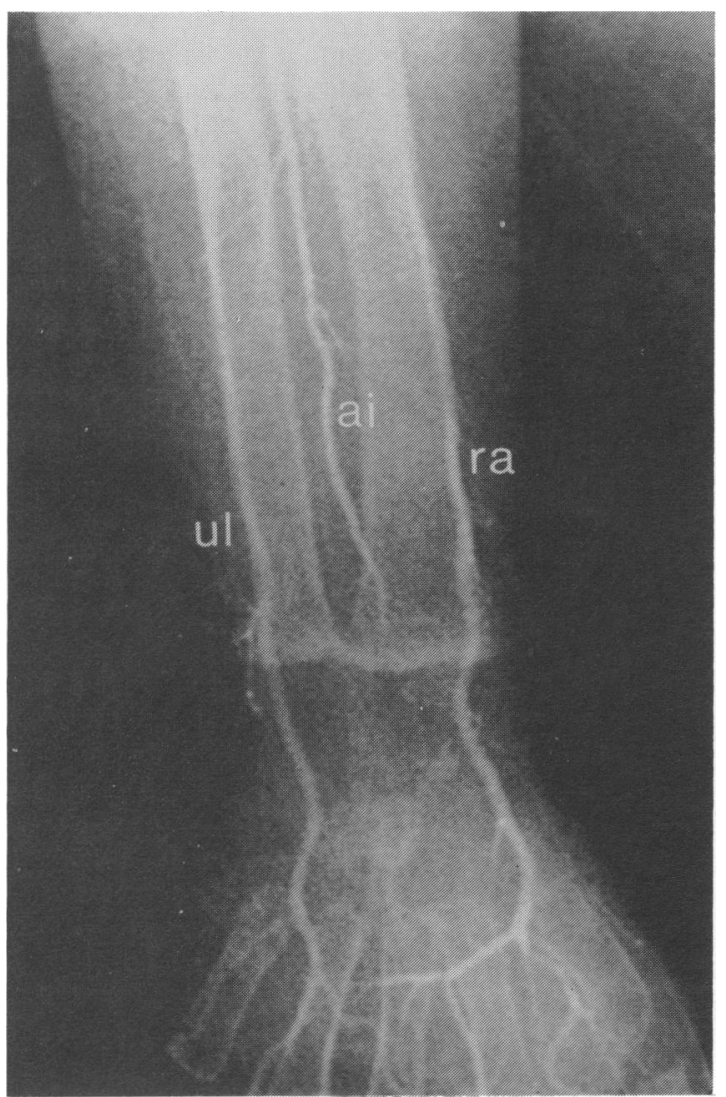

Fig. 1 Normal arterial pattern of the forearm. $r a=$ Radial artery, ai=anterior interosseous artery, and $u l=u l n a r$ artery. cardiac surgery for monitoring of blood pressure. This method was also used in another patient with clinically weak radial artery pulsations and normal heart for whom cardiac catherisation was not performed. Written consent was obtained from the parents after full explanation of the procedure. In both subclavian and brachial approaches meglumine iothalamate (Conray 280 ) $0.5 \mathrm{ml} / \mathrm{kg}$ body weight was injected by hand at $1-2 \mathrm{ml} / \mathrm{sec}$, and the arteriogram was recorded with cinematography. A subclavian arteriogram was performed during cardiac catheterisation in 10 patients with congenital heart disease but no clinical abnormalities of the radial or ulnar artery pulses for comparison.

\section{Results}

In none of the normal children with or without congenital heart disease was an abnormal artery detected clinically. On the other hand, 12 patients $(16 \%)$ with Down's syndrome were found to have abnormal arteries in the wrist. Nine were seen

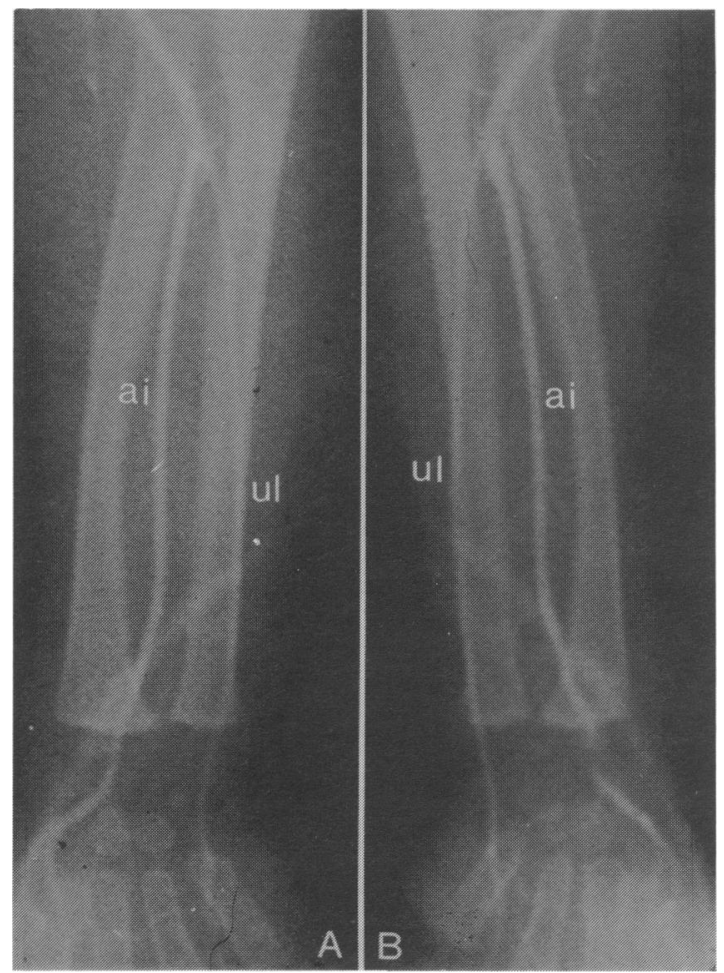

Fig. 2 Right $(A)$ and left $(B)$ forearm of patient with absent radial artery and enlarged anterior interosseous artery (ai). ul=Ulnar artery. 
among the 50 cases $(18 \%)$ with congenital heart disease and three among the $27(11 \%)$ with normal hearts; there was no statistical difference between the two groups $\left(\chi^{2}\right.$ test). Girls were predominantly affected, comprising 10 of the 12 cases $(0.05>p>0.01)$. The Table lists the nature of the arterial abnormalities detected and the associated congenital heart diseases.

Figure 1 shows the normal arterial pattern in the forearm in patients with normal radial and ulnar pulsations in the wrist. The radial artery is larger than the ulnar artery and the anterior interosseous artery is a relatively small artery that takes its origin from the ulnar artery and tapers towards the wrist.

Four types of clinical-angiographic abnormalities of the forearm arteries were observed in patients with Down's syndrome. These were as follows.
(1) The most commonly encountered abnormality was an absent radial artery pulsation in the usual position over the wrist but presence of a strong arterial pulsation over the dorsum in the lowermost portion of the forearm, which could be followed caudally over the dorsal aspect of the wrist to enter the first dorsal interosseous muscle. This pattern was seen in seven of the 12 cases. Angiography showed absence of the radial artery and an enlarged anterior interosseous artery in these patients (Fig. 2).

(2) In one patient the dorsal artery was present together with the radial and ulnar arteries (Fig. 3). The radial artery, however, did not course round the wrist to the back of the hand as it usually behaves.

In both the above groups the dorsal artery represents the distal portion of the anterior interos-

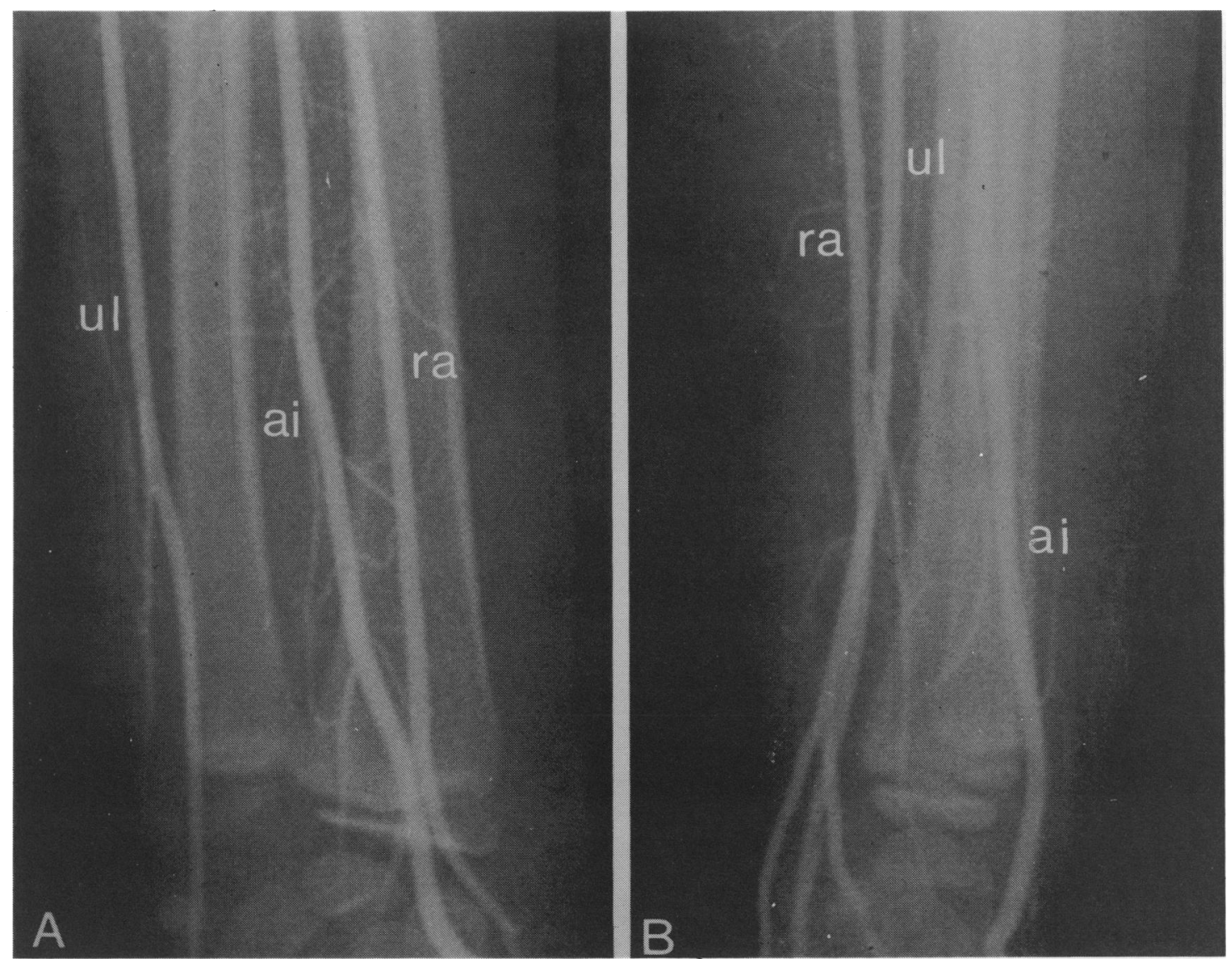

Fig. 3 Anteroposterior $(A)$ and lateral $(B)$ view of patient with large anterior interosseous artery (ai) present together with the radial (ra) and ulnar (ul) arteries. The anterior interosseous artery appears on the back of the hand instead of the radial artery. 
seous artery, which runs down the flexor surface of the forearm and, when near the wrist, turns dorsally to appear over the back of the hand where it takes over the function of the radial artery (Fig. 3 (B)).

(3) Three patients had a weak radial artery pulsation in the normal position and a strong ulnar artery pulsation. Angiography in two confirmed a small radial and a relatively large ulnar artery (Fig. 4).

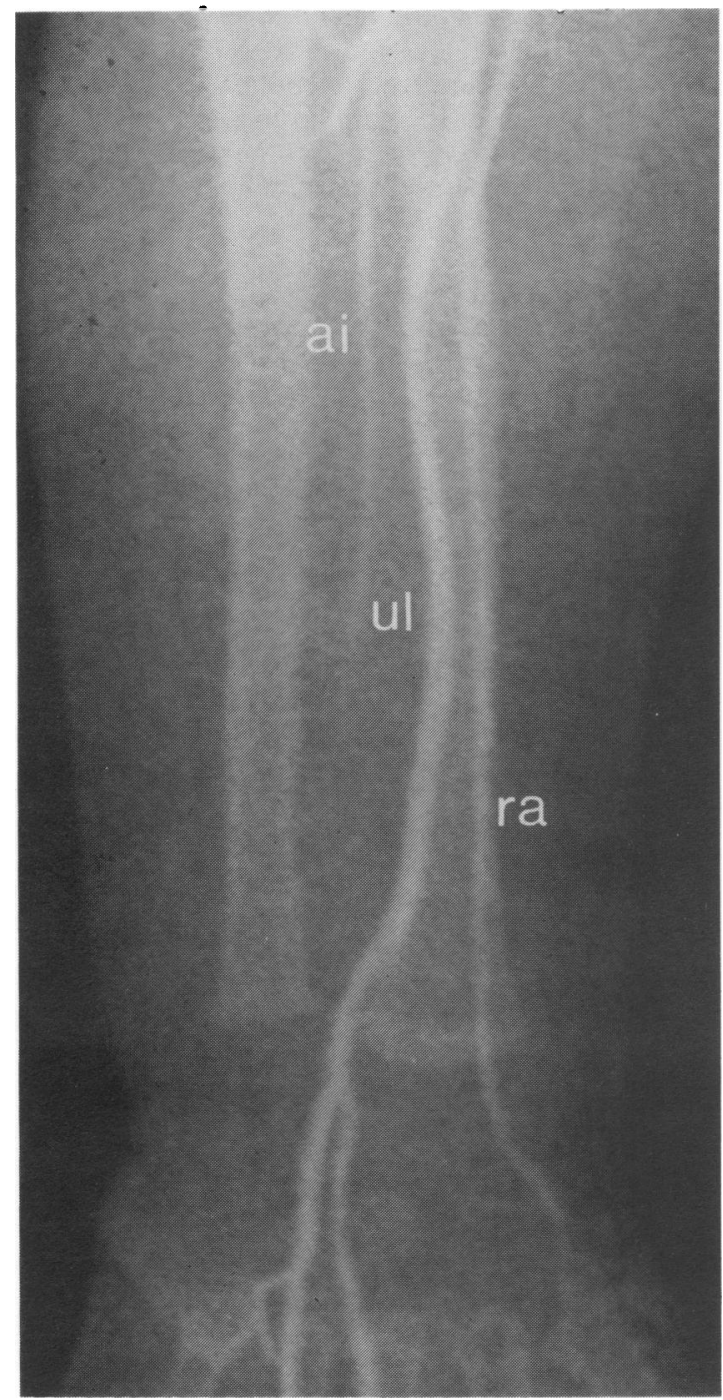

Fig. 4 Small radial (ra) and large ulnar (ul) artery in one patient. Note rather lateral course of the ulnar artery in the forearm in this patient. ai=Anterior interosseous artery.
(4) In one patient the radial artery was absent and was not replaced with a dorsal artery. A brachial arteriogram showed a vestigial radial artery, which ends as fine muscular branches in the forearm (Fig. $5)$. The anterior interosseous artery was not hypertrophied.

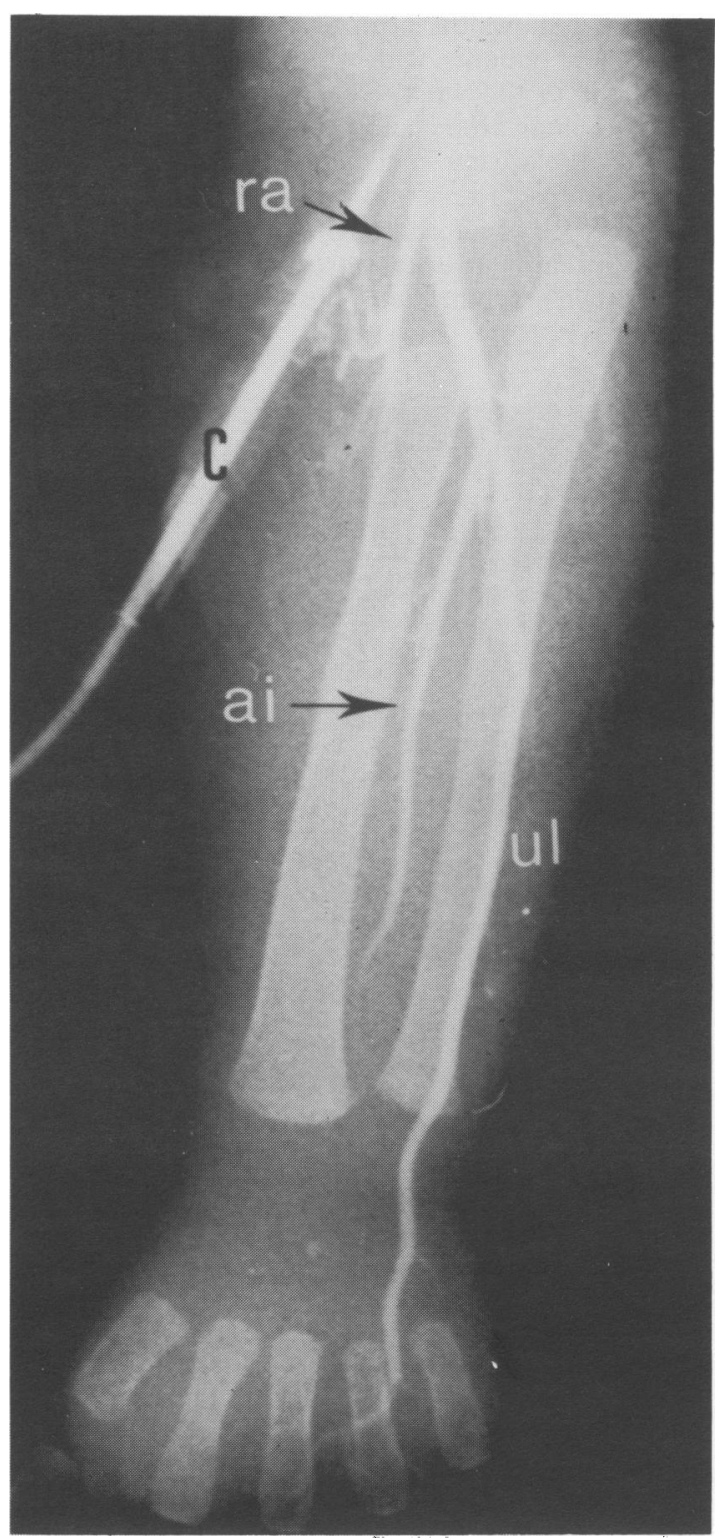

Fig. 5 Forearm of patient with vestigial radial (ra) artery terminating in muscles of forearm. Normal ulnar (ul) and anterior interosseous (ai) artery. C=Cannula. 
The arterial abnormality was seen on both right and left hands in eight patients and was unilateral in four. When present on both sides the same type of abnormality was observed. There was no predilection for any particular type of congenital heart disease.

\section{Discussion}

Congenital heart disease is present in $40 \%$ (range $7-71 \%$ ) of patients with Down's syndrome, ${ }^{378}$ the commonest anomaly being an atrioventricular defect or ventricular septal defect. Abnormalities of the great vessels are uncommon. Coarctation of the aorta occurs in less than $1 \%$ of the congenital heart diseases seen in patients with Down's syndrome ${ }^{4-6}$ compared with $5-8 \%$ in normal children. ${ }^{78}$ An aberrant right subclavian artery originating from the descending aorta is probably more common in patients with Down's syndrome, with reported incidence varying from 2.5 to $37.5 \%$, whereas it occurs in less than $10 \%$ of normal children with congenital heart disease and $0.5 \%$ of the general population. ${ }^{910}$ This aberration was present in seven $(14 \%)$ of our 50 patients with Down's syndrome with abnormal hearts. Other congenital vascular anomalies have not been reported. We were the first to describe abnormalities of the radial artery in Down's syndrome, being present in $18 \%$ of those patients with congenital heart disease and in $11 \%$ of those with normal hearts.

The radial artery normally originates from the brachial artery in the antecubital fossa and runs between the brachioradialis muscle and the flexor digitorum sublimis and flexor pollicis longus muscles up to the wrist where it becomes palpable between the tendon of the flexor carpi radialis muscle and the lateral prominence of the radius. Just beyond the styloid process of the radius it curves to the back of the hand over the lateral ligaments of the wrist joint and underneath the tendons of the abductor pollicis longus and extensor pollicis bravis muscles. It then passes between the heads of the first dorsal interosseous muscle into the palm to form the deep palmar arterial arch. Fowler, in his classic text on cardiac diagnosis, has mentioned that impairment of the radial pulse is commonly caused by an aberrant course of the artery so that it is deep and not readily palpated. ${ }^{11}$ A deep lying, non-palpable radial artery has also been suggested by other authors on clinical examination of the cardiovascular system. ${ }^{12}$ Searching through the published reports on anatomy of the radial artery, however, yields no description of such an aberrant route of the radial artery in the forearm. The location of the ulnar artery pulsation in the wrist between the palmaris longus tendon and the pisiform bone is even more constant, and no deviation has been reported.

An abnormal course of the radial artery in the wrist running above instead of deep to the tendons of the abductor pollicis longus and extensor pollicis brevis on passing to the back of the hand has been described in five of 750 hands $(0.7 \%)$ by McCormack. ${ }^{13}$ Keen ${ }^{18}$ found three among 284 hands $(1 \%)$ in which the radial artery becomes superficial in the middle of the forearm, travelling across the surface of the brachioradialis muscle, and runs down in the superficial fascia to the wrist where it enters the first dorsal interosseous space in the usual manner. A superficial radial artery is also seen as a continuation of a 'superficial brachial artery', which takes its origin from the axillary artery or as the major branch of the brachial artery in the arm and travels superficially downwards. This superficial brachial and radial artery was present in $1.2 \%$ of McCormack's dissections. ${ }^{13}$

Eight of our patients had a superficial artery over the back of the wrist, but none of them seemed to bear the superficial or aberrant course of the radial artery described above. The dorsal artery seemed to emerge from beneath the extensor muscles in the distal forearm rather than across and over the brachioradialis muscle or the extensor tendons of the thumb. Angiographically, it seemed to be a direct continuation of the anterior interosseous artery, which travels between the radius and ulnar bones and then turns dorsally near the wrist to appear in the back of the hand. The radial artery was completely absent in seven and present in normal calibre in one patient. The anterior interosseous artery is indeed the most primitive axial vessel of the forearm and is the first to develop in the human embryo, in which it runs down the forearm and passes through the carpus to terminate on the dorsum of the hand. ${ }^{15}{ }^{19}$ This course of the vessel is still seen in the duck billed platypus (Ornithorhynchus). Thus it is reasonable to consider the abnormality seen in these patients as developmental absence of the radial artery and persistence of an embryologically dominant anterior interosseous artery, which in its distal portion takes over the function of the former.

Absence of the radial artery has hardly been reported. Charles and Chhatrapati have each described a case in which the lower end of the anterior interosseous artery took the course of radial at the wrist and into the hand as the deep palmar arterial arch. ${ }^{14} 15$ Not a single case was found in a total of 1750 dissections of the hand carried out by McCormack, Coleman, and Motamed. ${ }^{13} 1617$ Our patient with vestigial radial artery resembles the case described by Chhatrapati, whose dissection showed 
the brachial artery divided into a larger branch, which was the ulnar artery, and a smaller branch, which was thin and short and ended in the substance of the brachioradialis muscle. Diminutive as the artery was in both cases, it should in fact represent a partially developed rather than absent radial artery.

Three of our patients showed a weak radial and a relatively strong ulnar artery pulsation, which was confirmed by a larger ulnar artery on angiography. A large radial artery in the human being is an evolutionary achievement in the development of the hand. In lower primates like the apes and gibbons, which are adept at climbing, supination and pronation of the hand are fairly free. The ulnar artery, being on the fixed side of the forearm, is the larger vessel, while the radial artery is generally smaller because constant rotatory movements of the radius will not facilitate flow through this vessel. In baboons, on the other hand, with their quadrupedal walking behaviour and less twisting movements of the wrist, the radial artery is larger than the ulnar. In the intermediate simian primates the two vessels are equal in size. ${ }^{19}$

The four types of abnormalities of the arteries in the forearm observed in our 12 patients seem to represent a range of developmental primitiveness in the formation of the arteries of the forearm in Down's syndrome. The anterior interosseous artery remains as the dominant vessel of the forearm when the radial artery fails to develop. In a single patient the anterior interosseous artery has persisted and shared codominance with the radial and ulnar arteries at the wrist. A vestigial or small radial artery compared with the ulnar artery represents an arrest at different stages of its development.

Patients with Down's syndrome are more likely to have congenital heart diseases requiring surgical correction. Arterial cannulation for monitoring of the blood pressure is commonplace during the perioperative period. An abnormal radial artery in the wrist should be carefully looked for and cannulation of this vessel or the ulnar artery should best be avoided less circulation to the hand be compromised

\section{References}

1 Davies DV, Coupland RE. Gray's anatomy. 34th ed. London: Longmans, 1967:829-30.

${ }^{2}$ Romanes GJ. Cunningham's textbook of anatomy. 12th ed. Oxford: Oxford University Press, 1981:880-2.

${ }^{3}$ Greenwood DG, Nadas AS. The clinical course of cardiac disease in Down's syndrome. Pediatrics 1976;58:893-7.

4 Rowe RD, Uchida IA. Cardiac malformations in mongolism: prospective study of 184 mongoloid children. Am J Med 1961;31:726-35.

${ }^{5}$ Laursen HB. Congenital heart disease in Down's syndrome. $\mathrm{Br}$ Heart $J$ 1976;38:32-8.

${ }^{6}$ Park SC, Mathews RA, Zuberbuhler JR, et al. Down syndrome with congenital heart malformation. Am J Dis Child 1977;131:29-33.

7 Keith JD, Rowe RD, Vlad P. Heart disease in infancy and childhood. 3rd ed. New York: Macmillan Publishing Co, 1978.

${ }^{8}$ Nadas AS, Fyler DC. Pediatric cardiology. 2nd ed. Philadelphia: W B Saunders, 1972.

9 Goldstein WB. Aberrant right subclavian artery in mongolism. American Journal of Roentgenography 1965;95:13-6.

${ }^{10}$ Klinkhamer AC. Esophagography in anomalies of the aortic arch system. Amsterdam: Excerpta Medical Foundation, 1969.

11 Fowler NO. Cardiac diagnosis and treatment. 2nd ed. Maryland: Harper and Row Publishers, 1976:39.

12 Chamberlain EN, Ogilvie CM. Symptoms and signs in clinical medicine. Bristol: John Wright and Sons, 1967:154.

13 McCormack LJ, Cauldwell EW, Anson BJ. Brachial and antebrachial arterial patterns, a study of 750 extremities. Surg Gynecol Obstet 1953;96:43-54.

${ }^{14}$ Charles JJ. A case of absence of the radial artery. Journal of Anatomy and Physiology 1894;28:449.

15 Chhatrapati DN. Absence of radial artery. Indian J Med Sci 1964;18:462-5.

16 Coleman SS, Anson BJ. Arterial patterns in the hand based upon a study of 650 specimens. Surg Gynecol Obstet 1961;113:409-24.

17 Motamed HA. Anatomy, radiology and kinesiology of the hand unit. Chicago: Motamed Medical Publisher, 1982

${ }^{18}$ Keen JA. A study of the arterial variations in the limbs, with special reference to symmetry of vascular patterns. Am J Anat 1961;108:245-61.

19 Manners-Smith T. The limb arteries of primates. Journal of Anatomy and Physiology 1910;45:23-64.

Correspondence to Dr R N S Lo, Department of Pediatrics, Queen Mary Hospital, Pokfulam Road, Hong Kong.

Received 13 May 1986 